\title{
Umbilical Cord Blood Culture for Neonatal Sepsis: Do we Have an Answer?
}

\author{
Anu Thukral ${ }^{1}$
}

Received: 21 August 2020 / Accepted: 21 August 2020 / Published online: 29 August 2020

(C) Dr. K C Chaudhuri Foundation 2020

Umbilical cord blood is the first hematologic source from the neonate. Use of umbilical cord blood is not a new concept. It can be a useful diagnostic test for early onset sepsis, but its use still remains an uncommon practice.

The gold standard for neonatal sepsis is blood culture collected from the peripheral vein [1]. However, there is variability in blood culture sensitivity due to inadequate sample volume, administration of antibiotics prior to sample collection and administration of intrapartum antibiotics. In addition, peripheral vein culture is a painful procedure which requires skilled health care workers who need to dedicate quality time for sampling. Umbilical cord blood on the other hand does not involve pain infliction, avoids iatrogenic stress and procedural complications.

Previous studies have suggested safety and reliability of umbilical cord blood culture (UCBC) for sepsis evaluation in asymptomatic term infants [2] and for universal screening of early onset sepsis in newborns with maternal risk factors [3]. Increased positivity on $\operatorname{UCBC}(\sim 20$ to $47 \%)$ in high risk newborns has also been demonstrated $[4,5]$.

The present prospective observational study on 80 inborn neonates published in this issue of IJP included newborns with birth weight $\geq 1000 \mathrm{~g}$ and gestational age $\geq 28$ wk with at least two risk factors for sepsis. The sensitivity, specificity, positive predictive value and negative predictive value for UCBC were $66.7 \%, 89.2 \%, 58.8 \%$ and $92.1 \%$; respectively assuming peripheral vein culture as the gold standard [4]. The authors highlight the lack of a uniform guideline as the rationale for the current study. There is actually no dearth of literature evaluating umbilical sampling for culture with peripheral vein blood culture both in developing and developed world

Anu Thukral

dranuthukral@gmail.com

1 Department of Pediatrics, All India Institute of Medical Sciences, New Delhi, India and the organism profile in the two samples has also been well studied. The present study reiterates the same results [6].

Umbilical sampling is technically less challenging. Moreover it ensures adequate volume of culture. It can be obtained at an earliest possible time, which facilitates rapid initiation of antibiotics. It decreases the chances of anemia of prematurity and thus decreases blood transfusion related complications; but it has more chances of contamination from maternal vaginal and skin floras. However, proper aseptic preparation of the umbilical cord can eliminate this issue.

Despite ample evidence of umbilical cord blood being a beneficial alternative it has still not been adopted. The most important strategy is not another study but pooling data from existing literature and interprofessional collaboration amongst the neonatal and obstetric team and laboratory providers. In addition, a structured UCBC procedure is what is imperative. A premade kit with clear role allocation, checklist and clear labelling on the sample can decrease contamination risk [7]. In addition, the technique is important to obtain quality samples and hence periodic re-education of staff is required. Widespread adoption of this technology should be encouraged. Nevertheless, umbilical blood culture is not a substitution of a good bedside clinical monitoring of the neonate.

\section{Compliance with Ethical Standards}

Conflict of Interest None.

\section{References}

1. Dutta S, Kadam S, Saini SS, Bhakoo ON, Mathur NB; Writing group. Management of Neonatal Sepsis. Evidence based clinical practice guidelines. New Delhi: National Neonatology Forum of India; 2010. p. 155-72.

2. Hansen A, Forbes P, Buck R. Potential substitution of cord blood for infant blood in neonatal sepsis evaluation. Biol Neonate. 2005;88:12-8. 
3. Polin JI, Knox I, Baumgart S, Campman E, Mennuti MT, Polin RA. Use of umbilical cord blood culture for detection of neonatal bacteremia. Obstet Gynecol. 1981;57:233-7.

4. Kalathia MB, Shingala PA, Parmar PN, Parikh YN, Kalathia IM. Study of umbilical cord blood culture in diagnosis of early-onset sepsis among newborns with high-risk factors. J Clin Neonatol. 2013;2:169-72.

5. Pryles CV, Steg LN, Nair S, Gellis SS, Tenney B. A controlled study of the influence on the newborn of prolonged premature rupture of the amniotic membranes and/or infection in the mother. Paediatrics. 1963;31:608-2.
6. Meena R, Meena KK, Athwani V, Gothwal S, Bairwa GS, Sitaraman S. Umbilical cord blood culture in diagnosis of early onset neonatal sepsis. Indian J Pediatr. 2020. https://doi.org/10.1007/s12098-02003345-5.

7. Moore SP, Newberry DM, Jnah AJ. Use of placental/umbilical blood sampling for neonatal admission blood cultures: benefits, challenges, and strategies for implementation. Neonatal Netw. 2017;36:152-9.

Publisher's Note Springer Nature remains neutral with regard to jurisdictional claims in published maps and institutional affiliations. 